\title{
Appendix 1: Erstelltes Nameninventar kolonial motivierter Straßennamen
}

\begin{tabular}{|c|c|}
\hline Stadt & SN-Token \\
\hline Altena & Karl-Peters-Straße \\
\hline Altenburg & Lettow-Vorbeck-Straße, Samoaweg, Togoweg \\
\hline Amberg & Lüderitzplatz \\
\hline Asch [Aš] & Lüderitzstraße \\
\hline Bad Godesberg & Gerhard-Rohlfs-Straße, Karl-Peters-Straße \\
\hline Bautzen & Dr.-Peters-Straße, Lüderitzstraße, Wissmannstraße \\
\hline Berlin & $\begin{array}{l}\text { Afrikanische Straße, Damarastraße, Dualastraße, Gröbenufer, Guinea- } \\
\text { straße, Iltisstraße, Kameruner Straße, Kiautschoustraße, Kongostraße, } \\
\text { Lansstraße, Lüderitzstraße, Mohasistraße, Nachtigalplatz, } \\
\text { Otavistraße, Pekinger Platz, Petersallee, Sambesistraße, Samoastra- } \\
\text { ße, Sansibarstraße, Senegalstraße, Swakopmunder Straße, } \\
\text { Takustraße, Tangastraße, Togostraße, Transvaalstraße, Ugandastra- } \\
\text { ße, Usambarastraße, Windhuker Straße }\end{array}$ \\
\hline Bochum & Lüderitzstraße, Petersstraße, Wissmannstraße \\
\hline Bottrop & $\begin{array}{l}\text { Gustav-Nachtigal-Straße, Karl-Peters-Straße, Lüderitzstraße, Wiss- } \\
\text { mannstraße }\end{array}$ \\
\hline $\begin{array}{l}\text { Brandenburg an der } \\
\text { Havel }\end{array}$ & Gustav-Nachtigal-Straße \\
\hline Braunschweig & $\begin{array}{l}\text { Albert-Voigts-Weg, Carl-Peters-Straße, Hermann-Blumenau-Straße, } \\
\text { Kamerunstraße, Lettow-Vorbeck-Straße, Lüderitzstraße, Otto-Finsch- } \\
\text { Straße, Swakopmunder Straße, Togoweg, Windhuker Straße, Wiss- } \\
\text { mannstraße }\end{array}$ \\
\hline Bremen & $\begin{array}{l}\text { Dualaweg, Gerhard-Rohlfs-Straße, Kamerunstraße, Kribiweg, } \\
\text { Leutweinplatz, Leutweinstraße, Lüderitzbrücke, Lüderitzstraße, } \\
\text { Nachtigalstraße, Otavistraße, Südweststraße, Togoplatz, Togostraße, } \\
\text { Waterbergstraße, Windhukstraße, Wissmannstraße }\end{array}$ \\
\hline Breslau [Wroctaw] & $\begin{array}{l}\text { Apiastraße, Dualastraße, Heinrich-Schnee-Straße, Karl-Peters-Straße, } \\
\text { Lettow-Vorbeck-Straße, Lüderitzstraße, Samoastraße, Tangastraße, } \\
\text { Togostraße, Windhukstraße, Wissmannstraße }\end{array}$ \\
\hline Chemnitz & Kamerunstraße, Wissmannstraße \\
\hline Cuxhaven & $\begin{array}{l}\text { Carsten-Niebuhr-Straße, Lettow-Vorbeck-Straße, Leutweinstraße, } \\
\text { Lüderitzstraße, Tsingtaustraße, Wissmannstraße }\end{array}$ \\
\hline Danzig [Gdańsk] & $\begin{array}{l}\text { Carl-Peters-Straße, Lüderitzstraße, Gustav-Nachtigal-Straße, Von- } \\
\text { Wissmann-Straße }\end{array}$ \\
\hline
\end{tabular}

๑ Open Access. () 2021 Verena Ebert, publiziert von De Gruyter. (c))BY-ND Dieses Werk ist lizenziert unter der Creative Commons Attribution-NoDerivatives 4.0 International Lizenz. 


\begin{tabular}{|c|c|}
\hline Stadt & SN-Token \\
\hline Delmenhorst & $\begin{array}{l}\text { Karl-Peters-Straße, Lettow-Vorbeck-Straße, Leutweinstraße, } \\
\text { Lüderitzstraße, Nachtigalstraße, Vogelsangstraße, Wissmannstraße }\end{array}$ \\
\hline Dessau & Lüderitzweg, Nachtigalweg, Petersweg, Wissmannweg \\
\hline Dresden & $\begin{array}{l}\text { Godeffroystraße, Karl-Peters-Straße, Leutweinstraße, Lüderitzstraße, } \\
\text { Nachtigalstraße, Rohlfsstraße, Swakopmunder Straße, Windhuker } \\
\text { Straße, Wissmannstraße, Woermannstraße }\end{array}$ \\
\hline Duisburg & $\begin{array}{l}\text { Kameruner Pfad, Kameruner Straße, Lüderitzallee, Otavistraße, } \\
\text { Swakopmunder Pfad, Swakopmunder Straße, Windhuker Pfad, Wind- } \\
\text { huker Straße, Waterbergpfad, Waterbergstraße }\end{array}$ \\
\hline Düsseldorf & $\begin{array}{l}\text { Lüderitzstraße, Lüderitzstraße, Meyer-Waldeck-Straße, Meyer- } \\
\text { Waldeck-Straße, Petersstraße, Petersstraße, Sodenstraße, Sodenstra- } \\
\text { ße, Solfstraße, Solfstraße, Trothastraße, Wissmannstraße, Woerman- } \\
\text { nstraße, Woermannstraße }\end{array}$ \\
\hline Eilenburg & Dr.-Nachtigal-Straße, Dr.-Peters-Straße, Lüderitzstraße \\
\hline Erfurt & Wissmannstraße \\
\hline Essen & $\begin{array}{l}\text { Askaristraße, Gustav-Nachtigal-Straße, Hansemannstraße, Kamerun- } \\
\text { straße, Karl-Peters-Straße, Lüderitzwiese, Samoastraße, Südseestra- } \\
\text { ße, Tangabucht, Windhukweg, Woermannstraße }\end{array}$ \\
\hline Forst (Lausitz) & $\begin{array}{l}\text { Karl-Peters-Straße, Kamerunstraße, Lüderitzstraße, Nachtigalstraße, } \\
\text { Swakopmunder Straße, Togostraße, Tangastraße, Waterbergstraße, } \\
\text { Wissmannstraße, Woermannstraße }\end{array}$ \\
\hline Frankfurt am Main & $\begin{array}{l}\text { Lettow-Vorbeck-Straße, Neuguineaweg, Samoaweg, Tangastraße, } \\
\text { Togoweg }\end{array}$ \\
\hline Frankfurt/Oder & Wißmannstraße \\
\hline Gelsenkirchen & Tangastraße, Waterbergstraße, Windhukstraße \\
\hline Glatz [Kłodzko] & Lettow-Vorbeck-Straße \\
\hline Gleiwitz [Gliwice] & $\begin{array}{l}\text { Lettow-Vorbeck-Straße, Lüderitzstraße, Petersweg, Windhukstraße, } \\
\text { Wissmannstraße }\end{array}$ \\
\hline $\begin{array}{l}\text { Gmünd bzw. } \\
\text { Schwäbisch Gmünd }\end{array}$ & Lüderitzweg, Petersweg \\
\hline Gotha & Adolf-Lüderitz-Straße, Von Lettow-Vorbeck-Straße \\
\hline Hagen & Karl-Peters-Straße, Lettow-Vorbeck-Straße \\
\hline Hamburg & $\begin{array}{l}\text { Askaristieg, Carl-Peters-Weg, Daressalamstraße, Dominikweg, Emin- } \\
\text { Pascha-Straße, Erckertstraße, Estorffstraße, Gerhard-Rohlfs-Weg, } \\
\text { Gustav-Nachtigal-Damm, Kameruneck, Kamerunkai, Kamerunweg, } \\
\text { Kamerunstraße, Karl-Peters-Weg, Lüderitzweg, Lettow-Vorbeck- } \\
\text { Straße, Otawiweg, Ostafrikadamm, Somalikamp, Südwestkamp, Tan- } \\
\text { gastraße, Theodor-Weber-Reihe, Togokai, Togostraße, Togoweg, } \\
\text { Waterberg, Windhuker Stieg, Windhukkai, Windhukstraße, Wiss- } \\
\text { mannsweg, Wissmannsweg }\end{array}$ \\
\hline
\end{tabular}




\begin{tabular}{|c|c|}
\hline Stadt & SN-Token \\
\hline Hannover & $\begin{array}{l}\text { Am Carl-Peters-Platz, Carl-Peters-Platz, Kamerunstraße, Lettow- } \\
\text { Vorbeck-Allee, Nachtigalstraße, Ostafrikastraße, Rohlfsstraße, } \\
\text { Woermannstraße, Wissmannstraße }\end{array}$ \\
\hline Heilbronn & $\begin{array}{l}\text { Guineastraße, Kamerunstraße, Karl-Peters-Straße, Karolinenweg, } \\
\text { Lettow-Vorbeck-Straße, Lüderitzstraße, Samoastraße, Tangastraße, } \\
\text { Togostraße, Tsingtauer Straße, Windhuker Platz }\end{array}$ \\
\hline Herford & $\begin{array}{l}\text { Adolf-Lüderitz-Straße, Carl-Peters-Straße, Gustav-Nachtigal-Straße, } \\
\text { Hermann-von-Wissmann-Straße }\end{array}$ \\
\hline Hildesheim & Karl-Peters-Straße \\
\hline $\begin{array}{l}\text { Hindenburg 0.S. } \\
\text { [Zabrze] }\end{array}$ & Lüderitzstraße, Petersstraße, Togostraße \\
\hline Iglau [Jihlava] & Lüderitzgasse \\
\hline Iserlohn & Karl-Peters-Straße \\
\hline Kaiserslautern & Karl-Peters-Straße \\
\hline $\begin{array}{l}\text { Karlsbad [Karlovy } \\
\text { Vary] }\end{array}$ & Herrmann-von-Wissmann-Straße \\
\hline Karlsruhe & $\begin{array}{l}\text { Karl-Peters-Straße, Lettow-Vorbeck.Straße, Lüderitzstraße, Wiss- } \\
\text { mannstraße }\end{array}$ \\
\hline Kassel & $\begin{array}{l}\text { Lüderitzstraße, Togoplatz, Togostraße, Windhukstraße, Wissmann- } \\
\text { straße, Woermannstraße }\end{array}$ \\
\hline Kattowitz [Katowice] & Lettow-Vorbeck-Straße \\
\hline Kiel & $\begin{array}{l}\text { Carl-Peters-Straße, Lettow-Vorbeck-Straße, Lüderitzstraße, } \\
\text { Nachtigalstraße, Wissmannstraße, Woermannstraße }\end{array}$ \\
\hline Koblenz & $\begin{array}{l}\text { Gustav-Nachtigal-Straße, Karl-Peters-Straße, Lüderitzstraße, Wiss- } \\
\text { mannstraße, Woermannstraße }\end{array}$ \\
\hline Köln & $\begin{array}{l}\text { Carl-Peters-Straße, Gravenreuthstraße, Gustav-Nachtigal-Straße, Iltis- } \\
\text { straße, Lansstraße, Kamerunstraße, Lettow-Vorbeck-Straße, Lüderi- } \\
\text { tzstraße, Takuplatz, Takustraße, Tangastraße, Togostraße, Wissmann- } \\
\text { straße }\end{array}$ \\
\hline $\begin{array}{l}\text { Königsberg i. Pr. bzw. } \\
\text { Königsberg (Pr.) } \\
\text { [Kaliningrad] }\end{array}$ & $\begin{array}{l}\text { Karl-Peters-Straße, Liebertstraße, Leutweinstraße, Lüderitzstraße, } \\
\text { Rohlfsstraße, Woermannstraße, Wissmannstraße }\end{array}$ \\
\hline Königshütte [Chorzów] & $\begin{array}{l}\text { Kamerunstraße, Kolonialstraße, Lettow-Vorbeck-Straße, } \\
\text { Lüderitzstraße }\end{array}$ \\
\hline $\begin{array}{l}\text { Landsberg an der } \\
\text { Warte [Gorzów Wiel- } \\
\text { kopolski] }\end{array}$ & Lüderitzstraße, Nachtigalstraße, Petersstraße, Wissmannstraße \\
\hline Leipzig & $\begin{array}{l}\text { Lüderitzstraße, Swakopmunder Straße, Windhuker Straße, Waterberg } \\
\text { Straße, Wissmannstraße }\end{array}$ \\
\hline Leslau [Włocławek] & Karl-Peters-Straße \\
\hline
\end{tabular}




\begin{tabular}{|c|c|}
\hline Stadt & SN-Token \\
\hline $\begin{array}{l}\text { Lodz bzw. } \\
\text { Litzmannstadt [tódź] }\end{array}$ & $\begin{array}{l}\text { Archipelstraße, Askaristraße, Daressalamer Straße, Dattelweg, } \\
\text { Hererostraße, Kakaoweg, Kalaharistraße, Kamerunstraße, } \\
\text { Killimandscharostraße, Kokosweg, Koloniestraße, Korallenweg, } \\
\text { Lettow-Vorbeck-Straße, Lüderitzstraße, Neuguineastraße, } \\
\text { Njassastraße, Palmenweg, Perlmutterstraße, Riffstraße, Samoastraße, } \\
\text { Suaheliweg, Südoster Straße, Südwester Straße, Taifunstraße, } \\
\text { Tanganjikastraße, Tangaweg, Togostraße, Tropfenpfad, } \\
\text { Windhukstraße }\end{array}$ \\
\hline Lübeck & Karl-Peters-Straße, Lettow-Vorbeck-Straße, Lüderitzstraße \\
\hline Ludwigsburg & Lüderitzstraße, Karl-Peters-Straße \\
\hline Ludwigshafen am Rhein & Nachtigalstraße, Petersstraße, Wissmannstraße \\
\hline Lüneburg & Karl-Peters-Straße \\
\hline Lünen & Lettow-Vorbeck-Straße \\
\hline Magdeburg & $\begin{array}{l}\text { Dualaweg, Daressalamer Weg, Gustav-Nachtigal-Straße, Kameruner } \\
\text { Weg, Leutweinweg, Lomeweg, Massaiweg, Tangaweg, Togoweg, } \\
\text { Swakopmunder Weg, Windhuker Weg, Woermannstraße, } \\
\text { Kilimandscharoweg, Waterberg, Wissmannstraße }\end{array}$ \\
\hline Mannheim & $\begin{array}{l}\text { Karl-Peters-Straße, Gustav-Nachtigal-Straße, Leutweinstraße, } \\
\text { Lüderitzstraße, Wissmannstraße }\end{array}$ \\
\hline Merseburg & $\begin{array}{l}\text { Lüderitzstraße, Nachtigalweg, Petersstraße, Tangaweg, Togoweg, } \\
\text { Windhukweg, Wissmannweg, Woermannstraße }\end{array}$ \\
\hline Mühlheim an der Ruhr & Dr.-Karl-Peters-Straße, Lüderitzstraße, Von-Lettow-Vorbeck-Straße \\
\hline München & $\begin{array}{l}\text { Anechostraße, Askaripfad, Bennigsenstraße, Daressalamstraße, } \\
\text { Daressalamstraße, Dominikstraße, Dualastraße, Emin-Pascha-Platz, } \\
\text { Gröbenstraße, Großfriedrichsburger Straße, Groß-Nabas-Straße, } \\
\text { Iltisstraße, Kamerunplatz, Kameruner Straße, Karl-Peters-Straße, } \\
\text { Kibostraße, Leutweinstraße, Lüderitzstraße, Lomeweg, Nachtigalplatz, } \\
\text { Nachtigalstraße, Maerckerstraße, Nettelbeckstraße, Rohlfsstraße, } \\
\text { Sansibarplatz, Samoaplatz, Samoastraße, Sansibarstraße } \\
\text { Swakopmunder Straße, Taku-Fort-Straße, Tangastraße, Togostraße, } \\
\text { Togostraße, Tsingtauer Straße, Tsingtaustraße, Usambarastraße, Von- } \\
\text { Erkert-Platz, Von-Erkert-Straße, Von-Gravenreuth-Straße, Von- } \\
\text { Heydebreck-Straße, Von-Trotha-Straße, Waterbergstraße, Windhuker } \\
\text { Straße, Wissmannstraße }\end{array}$ \\
\hline München Gladbach & Lettow-Vorbeck-Straße \\
\hline Münster & Lüderitzweg, Woermannweg \\
\hline $\begin{array}{l}\text { Neustadt an der Haardt } \\
\text { bzw. Weinstraße }\end{array}$ & $\begin{array}{l}\text { Gustav-Nachtigal-Straße, Karl-Peters-Straße, Lüderitzstraße, Von- } \\
\text { Wissmann-Straße }\end{array}$ \\
\hline Nürnberg & Wissmannplatz, Wissmannstraße \\
\hline Oberhausen & $\begin{array}{l}\text { Karl-Peters-Straße, Leutweinstraße, Lüderitzstraße, Windhuker Straße, } \\
\text { Wissmannstraße }\end{array}$ \\
\hline
\end{tabular}




\begin{tabular}{|c|c|}
\hline Stadt & SN-Token \\
\hline Oldenburg & Tangastraße \\
\hline Oranienburg & $\begin{array}{l}\text { Dualastraße, Kamerunstraße, Lüderitzstraße, Otavistraße, Swakop- } \\
\text { munder Straße, Tabrastraße, Togostraße, Transvaalstraße, Windhuk- } \\
\text { straße }\end{array}$ \\
\hline Pabianitz [Pabianice] & $\begin{array}{l}\text { Kamerunstraße, Karl-Peters-Straße, Lettow-Vorbeck-Straße, } \\
\text { Lüderitzstraße, Nachtigalstraße, Samoastraße, Togostraße, } \\
\text { Wissmannstraße }\end{array}$ \\
\hline Pirmasens & Dr. Carl-Peters-Straße \\
\hline Pirna & Dr. Carl-Peters-Straße, Gustav-Nachtigal-Straße \\
\hline Posen [Poznań] & $\begin{array}{l}\text { Karl-Peters-Straße, Lüderitzstraße, Lettow-Vorbeck-Straße, } \\
\text { Nachtigalstraße, Wissmannstraße }\end{array}$ \\
\hline Ravensburg & Gustav-Nachtigal-Weg, Lüderitzweg, Petersweg \\
\hline Recklinghausen & $\begin{array}{l}\text { Karl-Peters-Straße, Gustav-Nachtigal-Straße, Lettow-Vorbeck-Straße, } \\
\text { Lüderitzstraße, Von-Wissmann-Straße }\end{array}$ \\
\hline Riesa & Dr.-Karl-Peters-Straße, Dr.-Nachtigal-Straße, Lüderitzstraße- \\
\hline Saalfeld & Carl-Peters-Platz, Lettow-Vorbeck-Straße \\
\hline Saarbrücken & Lüderitzstraße \\
\hline $\begin{array}{l}\text { Saarlouis bzw. Saar- } \\
\text { lautern }\end{array}$ & Von-Lettow-Vorbeck-Straße \\
\hline $\begin{array}{l}\text { Schweidnitz } \\
\text { [Świdnica] }\end{array}$ & Lüderitzufer \\
\hline Schweinfurt & Karl-Peters-Straße, Lüderitzstraße \\
\hline Siegburg & Dr. Karl-Peters-Straße \\
\hline Solingen & Lüderitzweg, Wissmannstraße \\
\hline Stettin [Szczecin] & $\begin{array}{l}\text { Kamerunstraße, Lüderitzlandstraße, Tangastraße, Togostraße, } \\
\text { Waterbergstraße, Windhuker Straße }\end{array}$ \\
\hline Stolberg (Rhld.) & Dr. Karl-Peters-Straße \\
\hline Stuttgart & $\begin{array}{l}\text { Deutsch-Ostafrika-Straße, Deutsch-Südwestafrika-Straße, Kameruner } \\
\text { Straße, Lüderitzstraße, Leutweinstraße, Neuguineastraße, Otavi- } \\
\text { straße, Petersstraße, Samoastraße, Südseestraße, Togostraße, Tanga- } \\
\text { straße, Tsingtauer Straße, Windhuker Straße, Wissmannstraße }\end{array}$ \\
\hline $\begin{array}{l}\text { Swinemünde } \\
\text { [Świnoujście] }\end{array}$ & Karl-Peters-Straße, Lüderitzstraße \\
\hline Tübingen & Eduard-Haber-Straße \\
\hline Völklingen & $\begin{array}{l}\text { Karl-Peters-Straße, Lettow-Vorbeck-Straße, Lüderitzstraße, } \\
\text { Nachtigalstraße, Wissmannstraße }\end{array}$ \\
\hline $\begin{array}{l}\text { Waldenburg } \\
\text { [Watbrzych] }\end{array}$ & Petersstraße, Wissmannstraße \\
\hline
\end{tabular}




\begin{tabular}{ll}
\hline Stadt & SN-Token \\
\hline Weimar & Lüderitzstraße, Petersstraße, Rohlfsstraße \\
Wiesbaden & Karl-Peters-Straße \\
Wolfenbüttel & Karl-Peters-Straße, Lüderitzstraße, Wissmannstraße \\
Wuppertal & Lettow-Vorbeck-Straße, Samoastraße, Windhukstraße \\
Zerbst/Anhalt & Karl-Peters-Straße \\
Zwickau & Karl-Peters-Straße, Lüderitzstraße \\
\hline
\end{tabular}

